\title{
La réalisation du /r/ en Afrique Centrale : Une première exploration du français des locuteurs basaaphones (Cameroun).
}

\author{
Fatima Hamlaoui $^{1 *}$, Milena Injac ${ }^{1}$, et Emmanuel-Moselly Makasso ${ }^{2}$ \\ ${ }^{1}$ University of Toronto, Department of French, Toronto, Canada \\ ${ }^{2}$ MinReSI, Yaoundé, Cameroun
}

\begin{abstract}
Résumé. La présente étude constitue une première exploration de la variété de français parlée par un sous-groupe de locuteurs camerounais, les basaaphones. L'examen perceptif de 511 occurrences de /r/ provenant de deux locuteurs (parole lue et parole semi-spontanée) montre que plusieurs variantes sont employées dépendamment du contexte phonétique. Le $\mathrm{R}$ apical $[\mathrm{r}]$ et le $\mathrm{R}$ dorsal $[\mathrm{b}]$ ont tendance à alterner en position prévocalique, faisant de [в] une variante forte dans cette variété de contact. Une vocalisation du $\mathrm{R}$ est observée en contexte postvocalique, et ce malgré le fait que le basaa autorise les syllabes (C) VC. Les groupes consonantiques $\mathrm{C}+\mathrm{R}$ favorisent quant à eux la variante apicale, quelle que soit leur position dans la syllabe. Enfin, l'examen des répétitions d'un même mot montre qu'une forme de conditionnement lexical pourrait également déterminer le choix de certaines variantes (notamment [ $\mathrm{b}]$ vs. [r] en position explosive).
\end{abstract}

\begin{abstract}
The realization of $/ \mathbf{r} /$ in Central Africa: A preliminary exploration of the French of Basaa speakers (Cameroon). The present study concentrates on the variety of French in contact with Basaa, a Bantu language (A43) spoken in Cameroon. Based on the perceptive examination of 511 occurrences of $/ \mathrm{r} /$ in read and semi-spontaneous speech, it shows that several realizations of this consonant alternate depending on the phonetic context. The apical $\mathrm{R}[\mathrm{r}]$ and the dorsal $\mathrm{R}[\mathrm{\textrm { }}]$ are both found in prevocalic position, making of $[\mathrm{b}]$ a strong variant in this dialect. A vocalization of $R$ is observed in postvocalic contexts despite the fact that Basaa allows (C)VC syllables and, interestingly, $\mathrm{C}+\mathrm{R}$ clusters favor the apical variant no matter their position with respect to the syllable nucleus. Finally, repetitions of the same words tend to indicate that a form of lexical conditioning might also be at play in determining the choice of variant (particularly [ $\mathrm{b}]$ vs. $[\mathrm{r}]$ in syllable onset).
\end{abstract}

* Corresponding author: f.hamlaoui@utoronto.ca 


\section{Introduction}

Le présent article s'intéresse à la réalisation du phonème /r/ dans la variété de français des locuteurs dont la langue maternelle est le basaa, une langue bantoue du Nord-Ouest, classée A43 par Guthrie (1948). Elle est parlée par approximativement 300000 personnes (Simons \& Fenning 2017) en Afrique Centrale, et plus précisément dans les régions Centre et Littoral du Cameroun (voir Figure 1).

Le français standard ou international se caractérise par un seul phonème $/ \mathrm{r} /$. Il fait partie des langues, relativement rares, dans lesquelles la réalisation $\mathrm{du} / \mathrm{r} /$ est principalement la consonne fricative uvulaire [ $\mathrm{s}$ (Wiese 2011). On trouve également la variante sourde de cette consonne, $[\chi]$, avant et après les consonnes sourdes. On sait cependant qu'à travers les langues, la réalisation $\mathrm{du} / \mathrm{r} /$ est un marqueur linguistique fort de distinctions régionales, sociales ou encore générationnelles (Wiese 2011, Scobbie 2006). Ceci est également le cas en français, où le $/ \mathrm{r} /$ peut présenter d'autres lieux et modes d'articulation. Ainsi, dans les variétés canadiennes du français, on trouve aussi bien des variétés dans lesquelles le /r/ est principalement une consonne fricative uvulaire que des variétés dans lesquelles il est principalement une consonne apicale ([r] ou « r roulé ») (Côté \& Saint-Amant Lamy 2012). D'après Valdman (1978: 55), les locuteurs antillais réalisent principalement la consonne fricative vélaire $[\gamma]$ ou sa version labialisée $\left[\gamma^{\mathrm{w}}\right]$. Enfin, les travaux portant sur des variétés ouest-africaines du français rapportent quant à eux une prédominance de la consonne apicale $[r]$, notamment chez les locuteurs originaires du Burkina Faso (locuteurs de mooré) et du Mali (locuteurs de bambara) (Boula de Mareuil \& Boutin 2011).

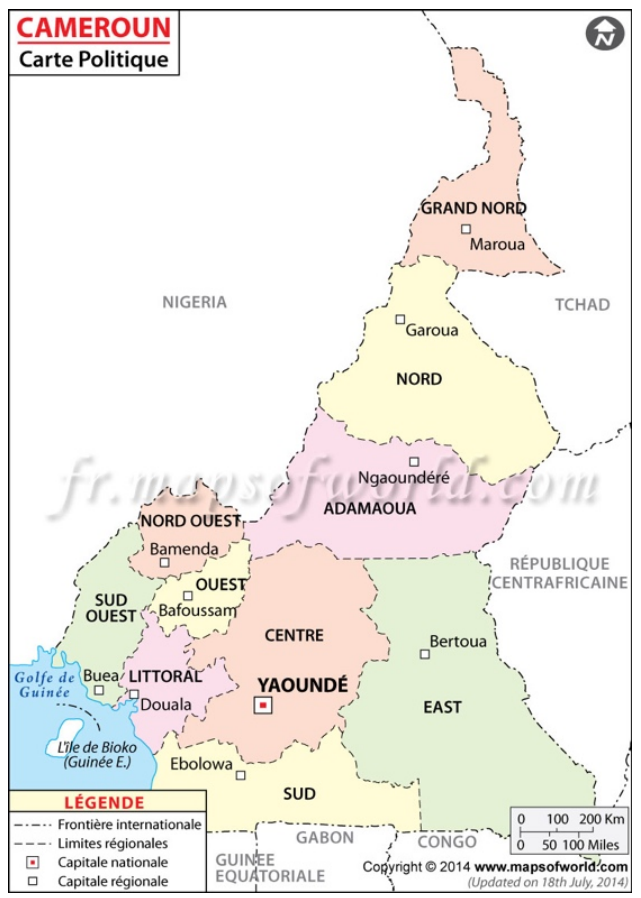

Fig. 1. Carte politique du Cameroun.

La présente étude porte sur deux corpus, l'un de parole lue, correspondant à un enregistrement audio du Dictionnaire Basaa-Français de Pierre Lemb \& François de Gastines (1973) et l'autre de parole semi-spontanée, correspondant à la traduction orale d'émissions de radio en basaa. Ces corpus sont extraits d'un corpus de parole plus large, d'environ 50 
heures de parole en basaa traduites oralement et aussi spontanément que possible en français par des locuteurs bilingues basaa-français (Hamlaoui et al. 2018). Ces données ont été collectées dans le cadre du projet BULB : Breaking the Unwritten Languages Barrier (Adda et al. 2016, Stüker et al. 2016).

Le basaa ne dispose pas pour sa part du phonème /r/. Dans cette langue, qui présente 22 phonèmes consonantiques (Hyman 2003), les consonnes battues alvéolaires [r] et [r] sont en effet des allophones du phonème /T/ (Makasso \& Lee 2015). On trouve par ailleurs la consonne fricative vélaire $[\gamma]$ et la consonne fricative uvulaire sourde $[\chi]$ comme allophones $\mathrm{du}$ phonème $/ \mathrm{K} /$. Les variantes voisées $[\mathrm{r}]$ et $[\mathrm{\gamma}]$ apparaissent dans les contextes intervocaliques et les variantes sourdes $[\delta]$ et $[\chi]$ devant les consonnes non-sonantes et en contexte pré-pausal. En basaa, la distribution et la réalisation des consonnes est contrainte par leur position au sein du mot et plus particulièrement au sein de la « racine prosodique ». Ainsi, d'après Hyman (2003), les 22 consonnes du basaa n'alternent réellement que dans la position initiale $(\mathrm{C} 1)$ de la racine prosodique, ainsi traitée comme une position forte. Dans les positions suivantes, soit C2, C3 et C4, on n'observe respectivement que 12, 6 et 3 des 22 consonnes.

Le basaa est particulièrement intéressant et se distingue de beaucoup d'autres langues, et particulièrement de langues maternelles ouest-africaines dont l'influence sur la réalisation du $/ \mathrm{r} /$ a été étudiée auparavant, en ce qu'il ne s'agit pas d'une langue CV (Boutin \& Turcsan 2009, Bordal 2009). Contrairement à la plupart des langues bantoues, le basaa est en effet une langue dans laquelle on trouve également des syllabes fermées de type (C)VC (Hyman 2003). Il s'agit donc de déterminer quelle(s) variante(s) de R sont employées, si le contexte phonétique a un effet sur le choix de la variante et si la L1 des basaaphones les amène à maintenir le /r/ en position de coda, là où des locuteurs venant de langues $\mathrm{CV}$ ont tendance à ne pas le réaliser (Lyche \& Skattum 2012).

Nos résultats constituent une première exploration de la variété de français parlée par un sous-groupe de locuteurs camerounais, les basaaphones. Ils montrent que plusieurs variantes sont employées et que le contexte phonétique joue en effet un rôle. Le $\mathrm{R}$ apical $[r]$ et le $\mathrm{R}$ dorsal $[\mathrm{s}$ ont tendance à alterner en position prévocalique. Un affaiblissement de la réalisation $\mathrm{du} / \mathrm{r} /$ est observé en contexte postvocalique, qui prend la forme d'une vocalisation $\mathrm{du} \mathrm{R}$ plutôt que d'une élision. Les groupes consonantiques ont quant à eux tendance à favoriser la variante apicale, quelle que soit leur position vis-à-vis du noyau de la syllabe. Ceci parait particulièrement intéressant en ce que d'autres variétés du français (Europe, Canada) ont tendance à élider le /r/ dans les groupes consonantiques finaux. Enfin, l'examen des répétitions d'un même mot montre qu'une forme de conditionnement lexical pourrait également déterminer le choix de certaines variantes (notamment $[\mathrm{r}] \mathrm{vs}$. [ $\mathrm{r}$ ] en position explosive). Nos résultats semblent donc jusqu'ici se distinguer de la variation libre observée par exemple par Boutin \& Turcsan (2009) en Côte d'Ivoire (au moins en contexte prévocalique) et se rapprocher plutôt de certaines des observations de Lyche \& Skattum (2012) pour certaines variétés de français parlées au Mali et Côté \& St-Amant Lamy (2012) pour le français de locuteurs de Trois-Rivières au Canada.

Le présent article est structuré comme suit. La Section 2 donne un bref aperçu des études antérieures concernant la réalisation $\mathrm{du} / \mathrm{r} /$ dans les variétés non-standards de français. La Section 3 présente notre corpus et notre méthodologie. La Section 4 se concentre sur nos résultats relatifs au conditionnement contextuel de la réalisation du /r/ dans la variété de français examinée. La Section 5 présente nos conclusions et perspectives.

\section{La réalisation du /r/ dans les variétés non-standards du français}

De nombreux travaux se sont intéressés à la réalisation $\mathrm{du} / \mathrm{r} /$ dans des variétés non-standards du français. Au-delà des différences de lieu d'articulation, ce qui semble rapprocher le plus les différentes variétés est la tendance $d u / r /$ à s'affaiblir en contexte implosif ou 
postvocalique, la tendance même qui aurait amené le français standard à passer progressivement du [r] (fort) au [в] (faible) au XVIIe siècle (Thomas 1986).

Ainsi, Valdman (1978 : 54) observe déjà que dans l'Océan Indien, le /r/ en position finale tend à s'affaiblir et à allonger la voyelle précédente, une tendance confirmée pour tous les créoles à base française par Nikiema (2002) et pour le créole réunionnais par Bordal (2006). Une tendance similaire est observée en Amérique du Nord, où le $\mathrm{R}$ vocalisé est en effet observé en position de coda (finale) chez les locuteurs de Montréal (Sankoff \& Blondeau 2010) et de Trois-Rivières (Côté \& St-Amant Lamy 2012). Il semblerait cependant que l'opposition entre une réalisation forte et une réalisation faible soit plus proche de celle impliquée dans l'évolution du français standard, soit s'articulant principalement autour d'un $\mathrm{R}$ apical en attaque et un $\mathrm{R}$ dorsal en coda (Clermont \& Cedergren 1971, Toussignant 1987).

Les travaux portant sur la réalisation $\mathrm{du} / \mathrm{r} /$ en Afrique subsaharienne semblent se concentrer particulièrement sur des variétés parlées en Afrique de l'Ouest et rapportent également une tendance à l'affaiblissement du /r/ en position implosive. Dans leur étude du français parlé au Mali, Lyche \& Skattum (2012) notent cependant que cette tendance est davantage prononcée chez les locuteurs dont la L1 est une langue CV : le bambara, le fulfulde et le syenara. Les locuteurs de L1 présentant des syllabes fermées (le songhay et le tamachek) tendent quant à eux à maintenir le /r/ en position de coda.

Avec plus de 300 langues nationales (Heine \& Nurse 2004) et ses deux langues officielles que sont le français et l'anglais, le Cameroun semble constituer un terrain privilégié pour explorer l'idée selon laquelle les propriétés phonotactiques de la L1 peuvent influencer la réalisation $\mathrm{du} / \mathrm{r} /$ en français. Notre échantillon de données est pour le moment limité, mais il vise à poser les bases d'une étude plus large de la réalisation $\mathrm{du} / \mathrm{r} /$ en français « camerounais ».

\section{Les données}

\subsection{Le corpus BULBasaa}

Les données examinées dans le présent article proviennent du corpus BULBasaa (Hamlaoui et al. 2018), un sous-ensemble des données collectées dans le projet BULB : Breaking the Unwritten Language Barrier (Adda et al. 2016, Stüker et al. 2016). L'objectif principal de ce projet est de développer des outils de traitement automatique du langage naturel $(N L P)$ visant à accélérer la documentation de langues non-écrites ou pour lesquelles peu de ressources textuelles sont disponibles. Le corpus BULBasaa comprend une cinquantaine d'heures de parole spontanée et contrôlée en basaa, dont une trentaine a déjà été oralement traduite en français par des locuteurs bilingues basaa-français. Ce corpus nous permet donc d'étudier le basaa aussi bien que la variété de français parlée par nos locuteurs bilingues. Les données examinées ici se limitent pour le moment à l'enregistrement du dictionnaire basaafrançais de Lemb \& De Gastines (1973), qui compte environ 6000 entrées accompagnées de leur traduction et de phrases illustrant leur emploi, et à la traduction, au pied levé, de deux émissions de radio en basaa. Il s'agit donc de parole lue et de parole semi-spontanée.

La locutrice ayant prêté sa voix à l'enregistrement du dictionnaire est originaire d'un village de la région Centre (Nyong et Kelle). Elle vit à Yaoundé et est âgée de 45 ans au moment des enregistrements (Été 2016). Sa langue maternelle est le basaa et elle a acquis le français à l'école à partir de l'âge de 4 ans, un contexte où seul le français était admis et où la variété parlée par son enseignante était probablement déjà influencée par la phonologie du basaa. Elle a suivi des études secondaires jusqu'au niveau de la Terminale et comme la majorité des Camerounais, elle emploie le français au quotidien dans ses échanges formels et comme langue véhiculaire avec les membres d'autres groupes linguistiques. Cette locutrice est particulièrement intéressante en ce qu'elle appartient à une génération qui, dans l'enfance, 
n'était exposée au français qu'à l'école. Elle se distingue ainsi de nombre de locuteurs plus jeunes qui avaient également accès au français standard ou hexagonal à travers les chaînes de télévision françaises et notamment les dessins animés. C'est notamment le cas de beaucoup de locuteurs grandissant actuellement en milieu urbain qui, désormais, acquièrent souvent le français en même temps ou avant le basaa.

Le locuteur ayant traduit les deux émissions de radio sur lesquelles nous nous concentrons ici est aussi originaire de la région Centre (Nyong et Kelle) et a grandi en milieu rural. Il vit également à Yaoundé et est âgé de 28 ans au moment des enregistrements (Été 2016). Sa langue maternelle est également le basaa et le français lui a été enseigné à l'école à partir de l'âge de 5 ans. Il a poursuivi des études supérieures et détient un doctorat au moment de l'enregistrement des données. Aucun de nos deux locuteurs n'a jamais séjourné en France.

Les deux locuteurs ont enregistré les données de façon autonome, à l'aide d'une tablette Samsung SM-T550 (Android) et de l'application LIG-Aikuma, une version améliorée de l'application Aikuma (Bird et al. 2014) développée dans le cadre du projet BULB (Blachon et al. 2016).

L'examen en cours de davantage de données, provenant d'autres types de locuteurs (âge, sexe, région d'origine etc.) nous permettra d'une part de déterminer dans quelle mesure les caractéristiques décrites dans le présent article sont généralisables à l'ensemble des locuteurs ayant le basaa pour L1, et d'autre, à quels facteurs sont dues les différences observées entre nos deux locuteurs.

\subsection{Notre méthodologie}

Nous avons examiné un total de 511 occurrences de /r/. Nous avons procédé à un codage perceptif et avons classé les différentes réalisations de R selon les 4 catégories suivantes : apical, dorsal, vocalisé et élidé. Les catégories distinguées sont semblables à celles des études antérieures en ce qu'elles se concentrent principalement sur le lieu d'articulation du /r/ (entre autres Boula de Mareuil \& Boutin 2011, Côté \& Saint-Amant Lamy 2012). Tout comme dans certaines des études antérieures, nous avons observé un taux élevé de vocalisation du/r/ dans certains contextes. Nous avons considéré, suivant Côté \& Saint-Amant Lamy (2012 : 1444), que les $\mathrm{R}$ vocalisés étaient ceux qui avaient tendance à allonger la voyelle précédente et «à l'écoute, ne comportaient aucune constriction consonantique.» Nous indiquons, dans le Tableau 1, le nombre $(\mathrm{N})$ et la proportion (\%) des différents types de $\mathrm{R}$ observés dans l'ensemble de données examinées. A travers l'ensemble des données examinées, la réalisation apicale $\mathrm{du} / \mathrm{r} /$ et sa réalisation dorsale sont presque aussi fréquentes, avec respectivement $42,5 \%$ et $40,9 \%$ des réalisations. Vient ensuite le $\mathrm{R}$ vocalisé, avec $15,3 \%$, et enfin le R élidé, avec seulement 1,4\% des réalisations.

Tableau 1. Réalisation de /r/ au sein de notre corpus.

\begin{tabular}{|l|c|c|c|c|c|c|c|c|c|c|}
\hline \multirow{2}{*}{ Corpus } & \multicolumn{2}{|c|}{ Apical } & \multicolumn{2}{c|}{ Dorsal } & \multicolumn{2}{c|}{ Vocalisé } & \multicolumn{2}{c|}{ Élidé } & \multicolumn{2}{c|}{ Total } \\
\cline { 2 - 12 } & $\mathrm{N}$ & $\%$ & $\mathrm{~N}$ & $\%$ & $\mathrm{~N}$ & $\%$ & $\mathrm{~N}$ & $\%$ & $\mathrm{~N}$ & $\%$ \\
\hline Dictionnaire & 142 & 48,8 & 71 & 24,4 & 71 & 24,4 & 7 & 2,4 & 291 & 100 \\
\hline Radio & 75 & 34 & 138 & 62,7 & 7 & 3,2 & 0 & 0 & 220 & 100 \\
\hline Total & 217 & 42,5 & 209 & 40,9 & 78 & 15,3 & 7 & 1,4 & 511 & 100 \\
\hline
\end{tabular}

Les résultats sont décrits en détails dans la section suivante. 


\section{Conditionnement contextuel}

Afin d'examiner l'effet du contexte syllabique sur la réalisation du /r/, nous nous sommes concentrés sur les contextes suivants :

- Contextes prévocaliques :

- Attaque simple en initiale de mot (par exemple : rejoindre)

- Attaque complexe $\mathrm{C}+\mathrm{R}$ (initiale ou interne au mot) (par exemple : écraser, crocodile)

- Attaque simple interne (par exemple : avarice, mesurer, barricader)

- Contextes postvocaliques :

- Coda simple en fin de mot (par exemple : jours, dire, cœur)

- Coda complexe $(\mathrm{R}+\mathrm{C})$ en fin de mot (par exemple : corde, ferme, sorte)

- Coda complexe $(\mathrm{C}+\mathrm{R})$ en fin de mot (par exemple: tendre, pauvre, palabre)

○ Coda simple interne (par exemple : tourner, murmurer, fourchu)

Dans le cas du $\mathrm{R}$ en attaque simple en initiale de mot, nous avons également distingué deux types : les mots contenant un seul $/ \mathrm{r}$ / et les mots contenant deux $/ \mathrm{r} /$. Nous souhaitions en effet déterminer si des effets d'assimilation étaient observés au sein d'un même mot.

\subsection{Contextes postvocaliques}

Comme dans l'étude de Côté \& Saint-Amant Lamy (2012), nous observons une proportion importante de vocalisation $\mathrm{du} / \mathrm{r} /$, notamment lorsque celui-ci se trouve en position de coda.

Sur 30 occurrences en coda simple en fin de mot, la vocalisation du $/ \mathrm{r} /$ est en effet observée dans $56,7 \%$ des cas dans nos données lues (dictionnaire). Tel que visible dans le Tableau 2, dans ce contexte notre locutrice réalise également un nombre important de $\mathrm{R}$ dorsaux $(26,7 \%)$. Le R apical ne représente quant à lui qu'une seule occurrence.

Tableau 2. Réalisation de /r/ en coda simple (fin de mot).

\begin{tabular}{|c|c|c|c|c|c|}
\hline Corpus & Apical & Dorsal & Vocalisé & Élidé & Total \\
\hline Dictionnaire & $1(3,3 \%)$ & $8(26,7 \%)$ & $17(56,7 \%)$ & $4(13,3 \%)$ & $30(100 \%)$ \\
\hline Radio & $2(6,5 \%)$ & $27(87,1 \%)$ & $2(6,5 \%)$ & $0(0 \%)$ & $31(100 \%)$ \\
\hline Total & $3(4,9 \%)$ & $35(57,4 \%)$ & $19(31,1 \%)$ & $4(6,5 \%)$ & $61(100 \%)$ \\
\hline
\end{tabular}

Notre locuteur produit quant à lui une vaste majorité de R dorsaux dans ce contexte $(87,1 \%)$ et ne présente que très peu de $\mathrm{R}$ vocalisés $(6,5 \%)$.

Plusieurs occurrences d'un même mot peuvent figurer dans les ensembles de données que nous avons examinés. Nous résumons la réalisation de ces occurrences dans le Tableau 3 pour le /r/ en coda simple (parole lue).

Tableau 3. Réalisation de /r/ en coda simple au sein d'un même mot (dictionnaire).

\begin{tabular}{|c|c|c|c|c|c|}
\hline Mot & Apical & Dorsal & Vocalisé & Élidé & Total \\
\hline pour & 0 & 3 & 3 & 0 & 6 \\
\hline sur & 1 & 0 & 3 & 3 & 7 \\
\hline unir & 0 & 1 & 3 & 0 & 4 \\
\hline tort & 0 & 1 & 2 & 0 & 3 \\
\hline
\end{tabular}

Dans tous les contextes où il figure au sein des entrées lexicales de notre dictionnaire, le mot « pour » se trouve en contexte pré-pausal, et dans trois cas, en position finale absolue (par exemple : raconter à, pour). L'alternance entre la réalisation dorsale du $/ \mathrm{r} /$ et sa réalisation 
vocalisée semble ici libre. Il en est de même pour les mots « sur », « unir » et « tort », qui peuvent être réalisés différemment dans un même contexte.

$\mathrm{Au}$ sein d'une coda complexe $(\mathrm{R}+\mathrm{C})$, la réalisation prédominante $\mathrm{du} / \mathrm{r} /$ pour notre locutrice est également vocalisée et dans une proportion encore plus grande que dans le cas $\mathrm{du} / \mathrm{r} /$ en coda simple $(76,7 \%)$. Tel qu'indiqué dans le Tableau 4, dans cette position, nous n'observons en effet que $23,3 \%$ de $\mathrm{R}$ dorsaux et aucun R apical ou élidé.

Tableau 4. Réalisation de /r/ en coda complexe $\mathrm{R}+\mathrm{C}$ (fin de mot).

\begin{tabular}{|c|c|c|c|c|c|}
\hline Corpus & Apical & Dorsal & Vocalisé & Élidé & Total \\
\hline Dictionnaire & $0(0 \%)$ & $7(23,3 \%)$ & $23(76,7 \%)$ & $0(0 \%)$ & $30(100 \%)$ \\
\hline Radio & $3(11,1 \%)$ & $23(85,2 \%)$ & $1(3,7 \%)$ & $0(0 \%)$ & $27(100 \%)$ \\
\hline Total & $3(5,3 \%)$ & $30(52,6 \%)$ & $24(42,1 \%)$ & $0(0 \%)$ & $57(100 \%)$ \\
\hline
\end{tabular}

Ces réalisations contrastent de nouveau avec ce qui est observé chez notre locuteur, qui réalise de nouveau principalement des $\mathrm{R}$ dorsaux $(85,2 \%)$. Bien qu'il réalise également des $\mathrm{R}$ n'appartenant pas au français standard (11,1\% de $\mathrm{R}$ apicaux et $3,7 \%$ de $\mathrm{R}$ vocalisés), ses productions paraissent s'en rapprocher davantage.

Lorsque plusieurs répétitions d'un même mot sont examinées, notre locutrice semble alterner librement entre les deux variantes observées dans cette position, comme on peut le voir dans le Tableau 5, qui présente les différentes réalisations du /r/ au sein d'un même mot.

Tableau 5. Réalisation de /r/ en coda complexe $\mathrm{R}+\mathrm{C}$ au sein d'un même mot (dictionnaire).

\begin{tabular}{|c|c|c|c|c|c|}
\hline Mot & Apical & Dorsal & Vocalisé & Élidé & Total \\
\hline écorce & 0 & 0 & 2 & 0 & 2 \\
\hline force & 0 & 0 & 3 & 0 & 3 \\
\hline herbe & 0 & 1 & 1 & 0 & 2 \\
\hline marche & 0 & 0 & 3 & 0 & 3 \\
\hline porte & 0 & 0 & 3 & 0 & 3 \\
\hline sorte & 0 & 1 & 5 & 0 & 6 \\
\hline source & 0 & 0 & 2 & 0 & 2 \\
\hline
\end{tabular}

En position de coda interne au mot, la réalisation la plus fréquente de $/ \mathrm{r} /$ pour notre locutrice est, comme dans les deux contextes précédents, le R vocalisé (70\%). Dans cette position, le R apical est également relativement fréquemment observé (20\%). Le R dorsal et l'élision du /r/ sont quant à eux relativement rares, tel que l'illustre le Tableau 6.

Tableau 6. Réalisation de /r/ en coda (interne).

\begin{tabular}{|c|c|c|c|c|c|}
\hline Corpus & Apical & Dorsal & Vocalisé & Élidé & Total \\
\hline Dictionnaire & $6(20 \%)$ & $1(3,3 \%)$ & $21(70 \%)$ & $2(6,7 \%)$ & $30(100 \%)$ \\
\hline Radio & $3(10 \%)$ & $23(76,6 \%)$ & $4(13,3 \%)$ & $0(0 \%)$ & $30(100 \%)$ \\
\hline Total & $9(15 \%)$ & $24(40 \%)$ & $25(41,6 \%)$ & $2(3,3 \%)$ & $60(100 \%)$ \\
\hline
\end{tabular}

Notre locuteur se distingue de nouveau, en réalisant davantage de $\mathrm{R}$ dorsaux dans ce contexte, avec $76,6 \%$ des réalisations. Il produit également davantage de $\mathrm{R}$ vocalisés que dans les deux contextes postvocaliques précédents, avec un total de $13,3 \%$ des réalisations.

Comme on peut le voir au Tableau 7, différentes réalisations de /r/ peuvent être observées au sein d'un même mot. 
Tableau 7. Réalisation de /r/ en coda (interne) au sein d'un même mot (dictionnaire).

\begin{tabular}{|c|c|c|c|c|c|}
\hline Mot & Apical & Dorsal & Vocalisé & Élidé & Total \\
\hline courber & 1 & 0 & 3 & 0 & 4 \\
\hline détourner & 1 & 0 & 1 & 0 & 2 \\
\hline murmure(r) & 0 & 0 & 2 & 0 & 2 \\
\hline retourner & 1 & 0 & 2 & 0 & 3 \\
\hline tourner & 2 & 0 & 2 & 0 & 4 \\
\hline transformer & 0 & 1 & 1 & 0 & 2 \\
\hline
\end{tabular}

Lorsqu'il se trouve au sein d'une coda complexe de type $\mathrm{C}+\mathrm{R}$, la réalisation du phonème $/ \mathrm{r}$ / qui prédomine chez notre locutrice est la consonne apicale $(76,7 \%)$ et contraste ainsi avec la tendance observée jusqu'ici. Tel qu'indiqué dans le Tableau 8, la variante vocalisée est relativement rare dans ce contexte, avec seulement $10 \%$ des réalisations de notre locutrice et moins fréquente que la variante dorsale $(13,3 \%)$. Enfin, aucun cas d'élision n'est observé.

Tableau 8. Réalisation de /r/ en coda complexe C+R (fin de mot).

\begin{tabular}{|c|c|c|c|c|c|}
\hline Corpus & Apical & Dorsal & Vocalisé & Élidé & Total \\
\hline Dictionnaire & $23(76,7 \%)$ & $4(13,3 \%)$ & $3(10 \%)$ & $0(0 \%)$ & $30(100 \%)$ \\
\hline Radio & $36(100 \%)$ & $0(0 \%)$ & $0(0 \%)$ & $0(0 \%)$ & $36(100 \%)$ \\
\hline Total & $59(89,4 \%)$ & $4(6,1 \%)$ & $3(4,5 \%)$ & $0(0 \%)$ & $66(100 \%)$ \\
\hline
\end{tabular}

Dans ce contexte, notre locuteur réalise quant à lui exclusivement un $\mathrm{R}$ apical. Cela nous parait particulièrement intéressant en ce que d'après les travaux portant sur d'autres dialectes du français, nous nous attendons à un affaiblissement du $\mathrm{R}$ dans les contextes postvocaliques, y compris dans les coda complexes. Typiquement, aussi bien le français parlé en Europe que le français québécois tendent à simplifier les consonnes liquides lorsqu'elles suivent une consonne occlusive (Dell 1980, Côté 1997, Côté \& Saint-Amant Lamy 2012). Nous reviendrons sur ce point dans la discussion des résultats. Cela pourrait selon nous indiquer que nos locuteurs ne traitent pas le $\mathrm{R}$ battu ([r]) comme une consonne liquide mais, en cohérence avec leur L1, dans laquelle il s'agit d'un allophone de /T/, et les propriétés de ce son, comme une consonne occlusive (voir par exemple Colantoni \& Steele 2005 sur la relation de différentes consonnes rhotiques avec la catégorie des liquides et le traitement possible du $\mathrm{R}$ battu comme une consonne occlusive en espagnol). Il est cependant à noter que pour les deux locuteurs, une proportion élevée de leurs réalisations présente ici un schwa final $(56,6 \%$ pour notre locutrice et $61,1 \%$ pour notre locuteur), indiquant que s'ils ne simplifient pas ces groupes consonantiques, ils ont peut-être tendance à les préférer en attaque qu'en coda.

Parmi les mots répétés plusieurs fois dans notre corpus, nous observons également de la variation, mais moins que dans le cas du /r/ en coda simple (Tableau 9).

Tableau 9. Réalisation de /r/ en coda complexe C+R au sein d'un même mot (dictionnaire).

\begin{tabular}{|c|c|c|c|c|c|}
\hline Mot & Apical & Dorsal & Vocalisé & Élidé & Total \\
\hline prendre & 2 & 0 & 1 & 0 & 3 \\
\hline autre & 11 & 1 & 0 & 0 & 12 \\
\hline répandre & 2 & 0 & 0 & 0 & 2 \\
\hline joindre & 2 & 0 & 0 & 0 & 2 \\
\hline palabre & 1 & 0 & 1 & 0 & 2 \\
\hline
\end{tabular}

En somme, en ce qui concerne la réalisation de /r/ lorsqu'il se trouve en position postvocalique, nous observons principalement, chez notre locutrice, ce qui a été caractérisé comme un affaiblissement de cette consonne. En effet, sur les 120 occurrences de $/ \mathrm{r} / \mathrm{en}$ 
contexte postvocalique, 64 correspondent chez notre locutrice à un $\mathrm{R}$ vocalisé $(53,3 \%), 20$ à un $\mathrm{R}$ dorsal $(16,6 \%)$ et 6 à un $\mathrm{R}$ élidé $(5 \%)$. Le $\mathrm{R}$ apical, qui est parfois considéré comme emblématique des variétés de français parlées en Afrique subsaharienne n'y apparait que dans $25 \%$ des cas, mais est clairement favorisé au sein des codas complexes $\mathrm{C}+\mathrm{R}$, quels que soient le voisement et le lieu d'articulation de la consonne précédente. La variante apicale de $/ \mathrm{r} /$ est également largement favorisée par notre locuteur dans les codas complexes $\mathrm{C}+\mathrm{R}$. Ceci est particulièrement intéressant en ce que dans tous les autres contextes postvocaliques c'est la variante dorsale qui l'emporte chez lui. Ainsi, sur les 124 occurrences de /r/ examinées pour ce locuteur, 73 correspondent à un $\mathrm{R}$ dorsal $(58,8 \%), 44$ à un $\mathrm{R}$ apical $(35,5 \%)$ et seulement 7 à un $\mathrm{R}$ vocalisé $(5,6 \%)$.

\subsection{Contextes prévocaliques}

En contexte prévocalique, nous observons principalement, chez notre locutrice, ce qui est considéré comme un renforcement du $\mathrm{R}$, avec davantage de $\mathrm{R}$ apicaux qu'en contexte postvocalique. Notre locuteur semble quant à lui de nouveau se rapprocher davantage du français standard, avec une préférence générale pour le $\mathrm{R}$ dorsal.

En position d'attaque simple (initiale de mot), la réalisation de $/ \mathrm{r} /$ alterne pour notre locutrice entre sa variante apicale et sa variante dorsale. Aucune vocalisation ou élision n'est en effet observée dans cette position, tel qu'on peut le voir au Tableau 10.

Tableau 10. Réalisation de /r/ en attaque simple (initiale de mot, un seul R).

\begin{tabular}{|c|c|c|c|c|c|}
\hline Corpus & Apical & Dorsal & Vocalisé & Élidé & Total \\
\hline Dictionnaire & $15(50 \%)$ & $15(50 \%)$ & $0(0 \%)$ & $0(0 \%)$ & $30(100 \%)$ \\
\hline Radio & $5(16,6 \%)$ & $25(83,3 \%)$ & $0(0 \%)$ & $0(0 \%)$ & $30(100 \%)$ \\
\hline Total & $20(33,3 \%)$ & $40(66,6 \%)$ & $0(0 \%)$ & $0(0 \%)$ & $60(100 \%)$ \\
\hline
\end{tabular}

Dans ce contexte, la variante la plus fréquemment observée chez notre locuteur est le R dorsal $(83,3 \%)$, suivie du R apical, avec seulement $16,6 \%$ des cas.

Lorsqu'on examine la réalisation $\mathrm{du} / \mathrm{r} /$ à travers les différentes répétitions d'un même mot par notre locutrice, on observe, tel qu'indiqué dans le Tableau 11, que chaque mot semble associé à une seule des deux variantes réalisées en position d'attaque simple et que la variation est donc plus limitée qu'en contexte postvocalique. Cela semble suggérer un conditionnement lexical dans la réalisation $\mathrm{du} / \mathrm{r} /$.

Tableau 11. Réalisation de / $\mathrm{r} /$ en attaque simple (initiale de mot, un seul R) au sein d'un même mot (dictionnaire).

\begin{tabular}{|c|c|c|c|c|c|}
\hline Mot & Apical & Dorsal & Vocalisé & Élidé & Total \\
\hline ramasser & 0 & 3 & 0 & 0 & 3 \\
\hline raconter & 4 & 0 & 0 & 0 & 4 \\
\hline refuser & 0 & 2 & 0 & 0 & 2 \\
\hline royaume & 0 & 2 & 0 & 0 & 2 \\
rester & 3 & 0 & 0 & 0 & 3 \\
\hline
\end{tabular}

Une tendance similaire est observée dans la traduction des émissions de radio, avec cependant quelques mots présentant les deux variantes employées par notre locuteur. Ceci est visible au Tableau 12. 
Tableau 12. Réalisation de /r/ en attaque simple (initiale de mot, un seul $\mathrm{R}$ ) au sein d'un même mot (radio).

\begin{tabular}{|c|c|c|c|c|c|}
\hline Mot & Apical & Dorsal & Vocalisé & Élidé & Total \\
\hline reçu(s) & 0 & 2 & 0 & 0 & 2 \\
\hline réunion & 1 & 1 & 0 & 0 & 2 \\
\hline remerciements & 2 & 0 & 0 & 0 & 2 \\
\hline révérends & 0 & 3 & 0 & 0 & 3 \\
\hline regret & 1 & 2 & 0 & 0 & 3 \\
\hline radio & 0 & 3 & 0 & 0 & 3 \\
\hline raison & 0 & 4 & 0 & 0 & 4 \\
\hline rendu & 0 & 2 & 0 & 0 & 2 \\
\hline
\end{tabular}

Afin de pousser davantage l'analyse des données provenant de l'enregistrement du dictionnaire, nous avons tenté de déterminer si la présence d'un second $/ \mathrm{r} /$ au sein du même mot pouvait influencer la réalisation d'un /r/ initial. Ce qu'on observe, dans le Tableau 13, dans les mots contenant 2 occurrences de $/ \mathrm{r} /$, la variante apicale est en effet plus fréquemment observée $(73,3 \%)$.

Tableau 13. Réalisation de /r/ en attaque simple (initiale de mot, deux R).

\begin{tabular}{|c|c|c|c|c|c|}
\hline Corpus & Apical & Dorsal & Vocalisé & Élidé & Total \\
\hline Dictionnaire & $44(73,3 \%)$ & $10(16,7 \%)$ & $5(8,3 \%)$ & $1(1,7 \%)$ & $60(100 \%)$ \\
\hline
\end{tabular}

Tel qu'indiqué en Tableau 14, les répétitions d'un même mot tendent également à être réalisées de manière identique mais dans une moindre mesure que dans le contexte décrit au Tableau 11.

Tableau 14. Réalisation de /r/ en attaque simple (initiale de mot, deux R) au sein d'un même mot (dictionnaire).

\begin{tabular}{|c|c|c|c|c|}
\hline Mot & Apical & Dorsal & Mixte & Total \\
\hline rabrouer & 4 & 0 & 0 & 4 \\
\hline rattraper & 1 & 1 & 1 & 3 \\
\hline rentrer & 2 & 1 & 1 & 4 \\
\hline rendre & 7 & 0 & 0 & 7 \\
\hline
\end{tabular}

A l'exception de « rendre », les mots figurant au Tableau 14 ont en commun que le second $/ \mathrm{r} /$ appartient à un groupe consonantique de type $\mathrm{C}+\mathrm{R}$ en position d'attaque. Comme on peut le voir dans le Tableau 15, ce contexte semble en effet favoriser la variante apicale, avec $80,4 \%$ des réalisation de notre locutrice et $69,4 \%$ des réalisations de notre locuteur.

Tableau 15. Réalisation de /r/ en attaque C+R (initiale ou interne).

\begin{tabular}{|c|c|c|c|c|c|}
\hline Corpus & Apical & Dorsal & Vocalisé & Élidé & Total \\
\hline Dictionnaire & $41(80,4 \%)$ & $8(15,7 \%)$ & $2(3,9 \%)$ & $0(0 \%)$ & $51(100 \%)$ \\
\hline Radio & $25(69,4 \%)$ & $11(30,6 \%)$ & $0(0 \%)$ & $0(0 \%)$ & $36(100 \%)$ \\
\hline Total & $66(75,9 \%)$ & $19(21,8 \%)$ & $2(2,3 \%)$ & $0(0 \%)$ & $87(100 \%)$ \\
\hline
\end{tabular}

Toutes les répétitions des mots figurant aux Tableau 16 et 17, mis à part le mot « créer » et « adressée », semblent de nouveau être associées à une seule variante de R. 
Tableau 16. Réalisation de /r/ en attaque $C+R$ (initiale ou interne) au sein d'un même mot (dictionnaire).

\begin{tabular}{|c|c|c|c|c|c|}
\hline Mot & Apical & Dorsal & Vocalisé & Élidé & Total \\
\hline écraser & 3 & 0 & 0 & 0 & 3 \\
\hline cracher & 2 & 0 & 0 & 0 & 2 \\
\hline créer & 3 & 1 & 0 & 0 & 4 \\
\hline prendre & 2 & 0 & 0 & 0 & 2 \\
\hline agrandi(r) & 3 & 0 & 0 & 0 & 3 \\
\hline croire & 2 & 0 & 0 & 0 & 2 \\
\hline griller/é & 3 & 0 & 0 & 0 & 3 \\
\hline
\end{tabular}

Tableau 17. Réalisation de /r/ en attaque $\mathrm{C}+\mathrm{R}$ (initiale ou interne) au sein d'un même mot (radio).

\begin{tabular}{|c|c|c|c|c|c|}
\hline Mot & Apical & Dorsal & Vocalisé & Élidé & Total \\
\hline problèmes & 0 & 3 & 0 & 0 & 3 \\
\hline prendre & 2 & 0 & 0 & 0 & 2 \\
\hline prendre & 2 & 0 & 0 & 0 & 2 \\
\hline grande & 0 & 2 & 0 & 0 & 2 \\
\hline programme(s) & 2 & 0 & 0 & 0 & 2 \\
\hline programme(s) & 2 & 0 & 0 & 0 & 0 \\
\hline adressée & 1 & 1 & 0 & 0 & 2 \\
\hline prière & 2 & 0 & 0 & 0 & 2 \\
\hline délivrance & 0 & 2 & 0 & 0 & 2 \\
\hline vendredi & 2 & 0 & 0 & 0 & 2 \\
\hline
\end{tabular}

Comme dans le cas de $/ \mathrm{r} /$ en contexte postvocalique, une des positions en contexte prévocalique semble faire exception à la tendance observée jusqu'ici. En effet, lorsque le $/ \mathrm{r}$ / se trouve en position d'attaque interne au mot (Tableau 18), la variante dorsale du $\mathrm{R}$ est légèrement plus fréquente que sa variante apicale chez notre locutrice, avec respectivement $60 \%$ et $40 \%$ des réalisations. La variante dorsale est encore une fois la plus fréquente chez notre locuteur, avec $96,6 \%$ des réalisations.

Tableau 18. Réalisation de /r/ en attaque simple (interne).

\begin{tabular}{|c|c|c|c|c|c|}
\hline Corpus & Apical & Dorsal & Vocalisé & Élidé & Total \\
\hline Dictionnaire & $12(40 \%)$ & $18(60 \%)$ & $0(0 \%)$ & $0(0 \%)$ & $30(100 \%)$ \\
\hline Radio & $1(3,3 \%)$ & $29(96,6 \%)$ & $0(0 \%)$ & $0(0 \%)$ & $30(100 \%)$ \\
\hline Total & $13(21,6 \%)$ & $47(78,3 \%)$ & $0(0 \%)$ & $0(0 \%)$ & $60(100 \%)$ \\
\hline
\end{tabular}

La variation observée au sein des répétitions d'un même mot, en Tableau 19, parait également légèrement plus importante que dans le cas $\mathrm{du} / \mathrm{r} /$ en attaque simple en position initiale de mot.

Tableau 19. Réalisation de /r/ en attaque simple (interne) au sein d'un même mot (dictionnaire).

\begin{tabular}{|c|c|c|c|c|c|}
\hline Mot & Apical & Dorsal & Vocalisé & Élidé & Total \\
\hline barrer & 3 & 2 & 0 & 0 & 5 \\
\hline embarrasser/é & 0 & 2 & 0 & 0 & 2 \\
\hline murmurer & 0 & 2 & 0 & 0 & 2 \\
\hline respirer/é & 1 & 1 & 0 & 0 & 2 \\
\hline retirer/é & 1 & 2 & 0 & 0 & 3 \\
\hline
\end{tabular}

En résumé, nous observons qu'en contexte prévocalique (en excluant les mots contenant deux R), la variante la plus fréquemment observée chez notre locutrice est la variante apicale, avec 68 occurrences sur un total de $111(61,3 \%)$. Cette variante est particulièrement favorisée 
au sein des attaques complexes $\mathrm{C}+\mathrm{R}$, où elle est observée dans $80,4 \%$ des cas. La variante dorsale, bien qu'elle apparaisse presque aussi fréquemment que le $\mathrm{R}$ apical en attaque simple, n'est réalisée que dans 41/111 (37\%) des cas au total. En ce qui concerne notre locuteur, la variante dorsale est favorisée dans tous les contextes prévocaliques, avec 65 occurrences sur un total de 96 (67,7\%), excepté le contexte C+R, dans lequel il réalise 69,4\% de R apicaux.

\section{Discussion et perspectives}

Dans les données de français examinées dans la présente étude et qui proviennent de locuteurs bilingues dont la langue maternelle est le basaa (langue bantoue A43), nous avons observé que la réalisation de /r/ semble, comme dans d'autres variétés, conditionnée par sa position au sein de la syllabe. En effet, en contexte prévocalique les variantes dorsale et apicale semblent favorisées par notre locutrice, alors qu'en position postvocalique, la variante vocalisée est la plus fréquemment observée. Ainsi, l'opposition entre une réalisation forte et une réalisation faible $\mathrm{du} / \mathrm{r} / \mathrm{semble}$ pour elle principalement s'articuler autour d'une réalisation consonantique en contexte explosif et une réalisation vocalique en contexte implosif. Bien qu'il soit historiquement traité comme la variante faible en français hexagonal et qu'il semble le demeurer dans certains dialectes de français canadien, le $\mathrm{R}$ dorsal semble ici davantage alterner avec le $\mathrm{R}$ apical et donc être associé à une réalisation forte.

Notre locuteur semble quant à lui se rapprocher davantage du français standard en ce qu'il ne présente que très peu de $\mathrm{R}$ vocalisés et que le $\mathrm{R}$ dorsal est la variante la plus fréquemment employée, aussi bien en contexte prévocalique qu'en contexte postvocalique.

Les groupes consonantiques $\mathrm{C}+\mathrm{R}$ semblent généralement faire exception, en ce qu'ils favorisent la variante apicale aussi bien en contexte prévocalique qu'en contexte postvocalique et ce pour nos deux locuteurs. Cela peut paraître étonnant en ce que dans d'autres dialectes du français, le /r/ est fréquemment élidé lorsqu'il appartient à une coda complexe (Dell 1980, Côté 1997). Ceci est particulièrement vrai lorsque la première consonne du groupe est une occlusive, ce qui peut s'expliquer par le fait que la suite occlusive + liquide (ou occlusive + fricative) constitue en position postvocalique une violation de la hiérarchie de sonorité (Côté 1997). Selon nous, le maintien du /r/ dans ce contexte peut peutêtre s'expliquer par la nature du $\mathrm{R}$ apical réalisé par nos locuteurs basaaphones, le $\mathrm{R}$ battu ([r]). Sur le plan phonétique, le R battu est en effet proche d'une occlusive (Ladefoged 2001). Sur le plan phonologique, [r] est dans la L1 de nos locuteurs un allophone de /T/. Cela pourrait également amener certains d'entre eux à traiter ce segment comme une consonne occlusive plutôt que comme une liquide tel que dans d'autres dialectes du français. La suite occlusive + occlusive ne transgressant pas la hiérarchie de sonorité (Côté 1997), cela pourrait expliquer le maintien et même la préférence pour cette variante dans les groupes consonantiques $C+R$ en position postvocalique. Nous avons cependant observé une tendance, plus marquée chez notre locuteur, à faire de ces groupes consonantiques des attaques par la réalisation du $e$ muet final. Davantage de recherches sont nécessaires pour comprendre comment le traitement des groupes consonantiques de cette variété de français est influencé par la L1 de nos locuteurs et se rapproche ou non d'autres variétés déjà bien décrites.

Enfin, bien que le basaa ne soit pas une langue CV, on constate également, notamment chez notre locutrice, un affaiblissement du $\mathrm{R}$ en coda (interne et finale), sans que celui-ci ne soit complètement élidé. Davantage de travaux semblent nécessaires pour déterminer si l'élision et la vocalisation s'opposent en effet en fonction des langues en contact avec le français et de leur structure syllabique. Tel que mentionné plus haut, il nous semble que la nature du R (c'est-à-dire roulé, battu etc.) et sa place dans la hiérarchie de sonorité pourrait peut-être également contribuer à expliquer pourquoi il est maintenu ou non en fonction des variétés de contact.

En ce qui concerne la variété de français en contact avec le basaa, l'examen en cours de davantage de données, notamment d'autres locuteurs, devrait nous permettre de confirmer si 
les tendances observées ici sont stables et de préciser les facteurs permettant d'expliquer les différences observées dans la présente étude.

\section{Références bibliographiques}

Adda, G., Stüker, S., Adda-Decker, M., Ambouroue, O., Besacier, L., Blachon, D., Bonneau-Maynard, H., Gauthier, E. Goddard, P., Hamlaoui, F., Idiatov, D., Kouarata, G.-N., Lamel, L., Makasso, E.M., Müller, M., Rialland, A., Van de Velde, M., Yvon, F., \& S. Zerbian. (2016). Breaking the Unwritten Language Barrier: The BULB Project. Procedia Computer Science, 81, 8-14.

Andzanga, R.S. (2017). Appropriation de la lecture et de l'écriture du français au Cameroun. Cas des Lycées bilingues de Yaoundé et de Buéa. Saint-Denis : Connaissances et Savoirs.

Bird, S., Hanke, F., Adams, O. \& H. Lee. (2014). Aikuma: A Mobile App for Collaborative Language Documentation. Proceedings of the 2014 Workshop on the Use of Computational Methods in the Study of Endangered Languages.

Blachon, D., Gauthier, E., Besacier, L., Kouarata, G.-N., Adda-Decker, M. \& A. Rialland. (2016). Parallel Speech Collection for Under-resourced Language Studies Using the LIG-Aikuma Mobile Device App. Procedia Computer Science, 81, 61-66.

Bordal, G. (2006). Traces de la créolisation dans un français régional : le cas du /R/ de l'île de la Réunion. Mémoire de Maîtrise, Université d'Oslo.

Bordal, G. (2009). A phonological study of French spoken by multilingual speakers from Bangui, the capital of the Central African Republic. In Durand, J., Laks, B. \& C. Lyche : Phonologie, variation et accents du français. Paris : Hermès, pp. 23-44.

Boula de Mareuil, P. \& B. A. Boutin. (2011). Evaluation et identification perceptives d'accents ouestafricains en français. French Language Studies, 21, 361-379.

Boutin, B. A. \& G. Turcsan. (2009). La prononciation du français en Afrique: la Côte d'Ivoire. In Durand, J., Laks, B. \& C. Lyche : Phonologie, variation et accents du français. Paris : Hermès, pp. 133-156.

Colantoni, L. \& J. Steele. (2005). Liquid asymmetries in French and Spanish. Toronto Working Papers in Linguistics 24, 1-14.

Côté, M.-H. (1997). Phonetic Salience and Consonant Cluster Simplification. In B. Bruening, Y. Kang \& M. McGinnis (eds.), PF: Papers at the Interface. MIT Working Papers in Linguistics 29. Cambridge, MA: MITWPL, pp. 229-262.

Côté, M.-H. \& H. Saint-Amant Lamy. (2012). D'un [r] à l'aut[s]e : contribution à la chute du R apical au Québec. In Neveu, F., Muni Toke, V, Blumenthal, P, Klingler, T., Ligas, P., Prévost, S. \& S. Teston-Bonnard: SHS Web of Conferences Volume 1, $3^{e}$ Congrès Mondial de Linguistique Française.

Dell, F. (1980). Generative Phonology and French Phonology. Translated from French by Catherine Cullen. Cambridge: Cambridge University Press.

Guthrie, M. (1948). The classification of the Bantu languages. London: Oxford.

Hamlaoui, F., E.-M. Makasso, M. Müller, J. Engelmann, G. Adda, A. Waibel \& S. Stüker. (2018). BULBasaa: A Bilingual Basaa-French Speech Corpus for the Evaluation of Language Documentation Tools. Proceedings of the $11^{\text {th }}$ International Conference on Language Resources and Evaluation.

Heine, B. \& D. Nurse. (2004). Les langues africaines. Paris : Karthala.

Hyman L. (2003). Basaa (A43). In D. Nurse \& G. Philippson: The Bantu Languages. London: Routledge.

Ladefoged, P. (2001). A Course in Phonetics. Fort Worth: Harcourt Brace College Publishers.

Lemb, P. \& F. De Gastines. (1973). Dictionnaire basaa-français. Douala : Collège Liberman. 
Lyche, C. \& I. Skattum. (2012). The phonological characteristics of French in Bamako, Mali. In R. Gess, C. Lyche \& T. Meisenburg: Phonological Variation in French: Illustrations from three continents. Amsterdam: John Benjamins, pp. 73-101.

Makasso, E.-M. \& S. Lee. (2015). Basaa (A43). JIPA, 45(1), 71-79.

Nikiema, E. (2002). Les (parlers) français ont le choix, les créoles ont l'r. In Bavoux, C. \& D. de Robillard : Créolistique et linguistique. Paris : Antrophos, pp77-96.

Sankoff, G. \& Blondeau, H. (2010). Instability of the $[\mathrm{r}] \sim[\mathrm{R}]$ alternation in Montreal French: the conditioning of a sound change in progress. In van de Velde, H., van Hout, R., Demolin, D. \& W. Zonnevelde: VaRiation: Sociogeographic, Phonetic and Phonological Characteristics of $/ \mathrm{r} /$. Amsterdam: John Benjamins.

Scobbie, J. M. (2006). R as a variable. In K. Brown: Encyclopedia of language and linguistics, Vol. 10. Amsterdam: Elsevier, pp. 337-344.

Simons, G. \& C. D. Fenning. (2017). Ethnologue: Languages of the world. Dallas: SIL International.

Stüker, S., Adda, G., Adda-Decker, M., Ambouroue, O., Besacier, L., Blachon, D., Bonneau-Maynard, H., Gauthier, E. Goddard, P., Hamlaoui, F., Idiatov, D., Kouarata, G.-N., Lamel, L., Makasso, E.M., Müller, M., Rialland, A., Van de Velde, M., Yvon, F., \& S. Zerbian. (2016). Innovative Technologies for Under-Resourced Language Documentation: The BULB Project. Proceedings of the $2^{\text {nd }}$ Workshop on Collaboration and Computing for Under-Resources Languages: 'Towards an Alliance for Digital Language Diversity'.

Thomas, A. (1986). La variation phonétique: cas du franco-ontarien. Ottawa : Didier.

Toussignant, C. (1987). La variation sociolinguistique. Modèles québécois et méthode d'analyse. Sillery : Presses de l’Université du Québec.

Valdman, A. (1978). Le Créole: structure, statut et origine. Paris : Klincksieck.

Wiese, R. (2011). The Representation of Rhotics. In van Oostendorp, M., Ewen, C., Hume, E. \& K. Rice: The Blackwell Companion to Phonology. New York: Blackwell. 